\title{
A Estruturação do Programa de Capacitação Profissional de Biossegurança no Contexto do Projeto de Modernização da Gestão Científica do Instituto Oswaldo Cruz
}

Development of a Biosafety Training Program Aligned with the Scientific Management Modernization Project of the

Maria Eveline de Castro Pereira

Mestre em Ciências. Membro da Comissão Interna de Biossegurança do Instituto Oswaldo Cruz/Fiocruz.

Endereço: Av. Brasil, 4365, Pavilhão Gomes de Faria, Sala 210, Manguinhos, CEP 21045-900, Rio de Janeiro, RJ, Brasil.

E-mail: maria®ioc.fiocruz.br

\section{Claudia Jurberg}

Doutora em Educação. Gestão e Difusão em Biociências pela UFRJ. Jornalista do Instituto Oswaldo Cruz/Fiocruz e da Universidade Federal do Rio de Janeiro/UFRJ.

Endereço: Av. Carlos Chagas, 373, Centro de Ciências da Saúde, bloco H, sala H200, CEP 21941-902, Rio de Janeiro, RJ, Brasil.

E-mail: cjurbergळbiogmed.ufrj.br

Maria de Nazaré C. Soeiro

Doutora em Biologia Celular e Molecular. Pesquisadora do Laboratório de Biologia Celular do Instituto Oswaldo Cruz/Fiocruz.

Endereço: Av. Brasil, 4365, Pavilhão Carlos Chagas, Térreo, Manguinhos, CEP 21045-900, Rio de Janeiro, RJ, Brasil.

E-mail: soeiro®ioc.fiocruz.br

\section{Cíntia M. Borba}

Doutora em Biologia Parasitária. Pesquisadora do Laboratório de Taxonomia, Bioquímica e Bioprospecção de Fungos do Instituto Oswaldo Cruz/Fiocruz.

Endereço: Av. Brasil, 4365, Pavilhão Leônidas Deane, 68 andar, sala 608, Manguinhos, CEP 21045-900, Rio de Janeiro, RJ, Brasil.

E-mail: cborba®ioc.fiocruz.br

\section{Resumo}

0 presente artigo apresenta as etapas de estruturação do Programa de Capacitação Profissional de Biossegurança (PCPB), em consonância com o Projeto de Modernização da Gestão Científica do Instituto Oswaldo Cruz (IOC), detalhando o ciclo planejamento-desenvolvimento-avaliação, em especial do Curso de Biossegurança em Laboratório de Pesquisa Biomédica. Inicialmente, para o ciclo diagnóstico foram aplicados questionários aos interlocutores dos laboratórios do IOC, os quais revelaram interesse de participação no PCPB para ambas as categorias profissionais (níveis médio e superior), indicando como temáticas preferenciais biossegurança e boas práticas de laboratório. $\mathrm{Na}$ fase de planejamento foi definido que o PCPB seria subdivido em dois projetos (Boas Práticas de Laboratório de Saúde Pública para os profissionais de nível médio e Curso de Biossegurança para Laboratórios de Pesquisa Biomédica para profissionais de nível superior). A seguir, na fase de estruturação do curso, os módulos contemplados incluíram: introdutório; riscos químico, físico e biológico; gestão da qualidade e experimentação animal. Assim, no período 2006-2008, foram capacitados 315 profissionais e realizadas avaliações segundo o modelo de David Kirkpatrick. O primeiro nível, chamado de reação, foi aferido e demonstrou que 54,03\% dos profissionais declararam que o curso foi excelente; $39,59 \%$ classificaram como bom e os demais $6,38 \%$ acharam que foi regular ou não opinaram. Para a 
avaliação do aprendizado foram realizados, a cada módulo, pré e pós-testes. Foi verificado que todos os módulos tiveram acréscimos nas médias do pósteste em relação ao pré-teste. Os resultados obtidos apontaram estratégias a serem seguidas no aperfeiçoamento desse modelo de educação continuada em biossegurança.

Palavras-chave: Biossegurança; Capacitação; Educação profissional; Boas práticas de laboratório

\section{Abstract}

In the present paper we report a Biosafety Training Program (BTP) developed at Oswaldo Cruz Institute (IOC/Fiocruz) aligned with the Scientific Management Modernization Project of IOC. The program, including the "Biosafety Course in Biomedical Research Laboratory", was structured according to the planning-development-evaluation cycle. Initially, for the diagnosis cycle, the IOC laboratories representatives answered a questionnaire that showed that both professional categories (middle and higher levels) were interested in participating in BTP and mentioned, as preferential themes, biosafety and good laboratory practices. In the planning phase it was defined that BTP would be divided into two projects (Good Laboratory Practices in Public Health for middle level professionals and Biosafety Course in Biomedical Research Laboratories for higher level professionals). During the development phase of the Biosafety Course, the following modules were chosen: introduction, chemical, physical and biological risks, quality management and animal experimentation. Thus, in the period from 2006 to 2008,315 professionals were trained and the respective evaluations were performed according to David Kirkpatrick's model. The first level of evaluation, called reaction, showed that $54.03 \%$ of the professionals said that the course was excellent, $39.59 \%$ classified the course as good and $6.38 \%$ as regular or did not express any opinion. For learning evaluation, pre and post-tests were carried out in each module. All the modules showed an increase in the grades of the post-test when compared to the pre-test. The results pointed to strategies that should be followed in order to improve this biosafety continuing education model.

Keywords: Biosafety; Training, Professional Education; Good Laboratory Practices. 


\section{Introdução}

A biossegurança (BS) é mais do que um conjunto de ações voltadas para prevenir e/ou minimizar os riscos inerentes às áreas consideradas de saúde - biologia, biomedicina, fisioterapia, fonoaudiologia, medicina, medicina veterinária, nutrição, odontologia, psicologia, serviço social e terapia ocupacional - pela Resolução 287/98 do Conselho Nacional de Saúde (Costa e Costa, 2007). A BS extrapola as ações relacionadas à área de saúde, por representar um conjunto de princípios, estratégias, diretrizes, procedimentos e saberes que contribui para a segurança das pessoas e para a qualidade dos serviços e produtos oferecidos (Pereira e col., 2009a). Adicionalmente, a BS pode estar diretamente relacionada a inúmeras atividades associadas às novas tecnologias químicas, radioativas e geneticamente engenheiradas (Porto e Freitas, 1997), áreas biotecnológicas (Marinho e col., 200o) e mesmo áreas agropecuárias (Barros e Silva, 2005).

Portanto, percebe-se que, na atualidade, a biossegurança é uma temática muito debatida pela sociedade, não só por sua natureza prática, uma vez que objetiva a preservação da saúde dos profissionais, da coletividade e do ambiente, mas também pela questão ética, tendo em vista que, em tempos de globalização, qualquer descuido ocorrido localmente pode se tornar uma ameaça generalizada, podendo chegar, inclusive, a países distantes (Labarthe e Pereira, 2008).

Quando se pretende implantar uma gestão de biossegurança não se deve perder de vista que o sucesso depende, em última instância, da aprendizagem de novas condutas, e que a velocidade e a qualidade nas quais se processará o aprendizado podem ser determinantes até mesmo para a sobrevivência de uma instituição. Segundo Amorim (1999), não é fácil alterar práticas de trabalhos arraigadas. Por outro lado, Mastroeni (2008) questiona a dificuldade da prática da biossegurança, destacando que a resposta pode estar condicionada a diversas categorias, como idade, cultura, responsabilidade e educação. Além disso, através de um levantamento detalhado dos agentes químicos, físicos e biológicos manipulados, das rotinas desenvolvidas, da tecnologia e infraestrutura disponíveis, torna-se possível avaliar com maior precisão o nível de contenção que definirá as ações de biossegurança específicas a serem adotadas em cada instituição. A contenção, segundo Pereira e colaboradores (2009b), integra principalmente três elementos: a prática e a técnica laboratorial (boas práticas ou boas condutas), os equipamentos de segurança (coletivo e/ou individual) e o projeto de contenção estrutural (a infraestrutura laboratorial).

É importante destacar a importância do desenvolvimento de expedientes para intensificar a capacidade das pessoas - e, por extensão, das instituições - de aprender. Aprender não implica, necessariamente, tornar sucata o conhecimento anterior, mas, sim, a possibilidade de adicionar o "novo" ao "velho" (Amorim, 1999). Nesse sentido, Fleury e Fleury (2006) ressaltam que a mudança de padrões, quando gradual, acontece de forma menos traumática, gerando menos ansiedade. Os novos valores, segundo os autores, são incorporados aos já existentes, ampliando o leque de alternativas para a solução de problemas.

Depresbiteris (2004) chama a atenção para o fato de que é insuficiente uma formação meramente empírica, de aprender fazendo, sem saber o "para que" e o "por que". O significado de "por que" fazemos uma coisa é elemento fundamental para o nosso melhor desempenho. É igualmente importante a avaliação constante da adesão às normas de biossegurança, bem como aperfeiçoamento e adaptações para atender às necessidades circunstanciais (Gir e col., 2004). Um exemplo desse dinamismo ocorreu em 2003, durante a epidemia da Síndrome Respiratória Aguda Grave (do inglês Severe Acute Respiratory Syndrome - SARS). A Fundação Oswaldo Cruz (Fiocruz) estabeleceu uma série de procedimentos visando desde o recebimento de amostras, até a realização de diagnósticos laboratoriais e atendimento clínico (Funasa, 2003).

Corroborando com esse pensamento, Mastroeni (2008) afirma que a educação é seguramente o único meio de modificarmos a cultura do fazer "fácil" ao invés de correto. Não basta construir laboratórios com equipamentos de última geração, sem investir em educação. Costa e colaboradores (2008) alertam que educação em biossegurança no Brasil, apesar de sua importância estratégica e social, ainda não 
está inserida nas diretrizes curriculares, nem no ensino público nem no privado. Sabe-se que a deficiência na formação profissional, em função do descompasso entre o ensino nas universidades e a prática, principalmente nas instituições de saúde, é um importante obstáculo no processo educativo. Por isso, é fundamental um programa de capacitação continuada no sentido de promover ações efetivas de proteção, privilegiando a biossegurança respaldada pelo senso de responsabilidade como atributo individual e não como uma prática imposta (Gir e col., 2004).

Com o intuito de formar profissionais capazes de refletir criticamente sobre a realidade organizacional, a Comissão Interna de Biossegurança do Instituto Oswaldo Cruz (CIBio/IOC), da Fundação Oswaldo Cruz (Fiocruz), no Rio de Janeiro, implementou em 2006 o "Programa de Capacitação Profissional em Biossegurança” (PCPB) em consonância com o Projeto de Modernização da Gestão Científica do Instituto Oswaldo Cruz, que apresenta como um dos principais objetivos o fortalecimento dos laboratórios, com gestão de projetos em redes temáticas e programas integrados, preservando a diversidade da pesquisa e serviços institucionais, com produção de conhecimento mensurável de acordo com os padrões internacionais, valorizando as parcerias públicoprivadas e assegurando disponibilização, integração e divulgação do conhecimento (IOC, 2007).

A compreensão do problema e as discussões sobre alternativas de ação, através do consenso, são os verdadeiros desafios de um Programa de Capacitação Profissional em Biossegurança (PCPB), pois práticas de trabalho enraizadas por anos de história não se deixam transformar rapidamente. 0 aprendizado depende da capacidade de respostas eficazes às transformações do meio (adaptabilidade). Por isso, é necessário contemplar ações voltadas para a qualidade e eficácia da comunicação, além de investimentos em recursos organizacionais (seja na infraestrutura básica dos laboratórios ou na aquisição de equipamentos de proteção individual e coletiva).

\section{Objetivo}

Este artigo objetiva descrever a experiência de implantação do Programa de Capacitação Profissional em Biossegurança (PCPB) no IOC, em especial do Curso de Biossegurança em Laboratório de Pesquisa Biomédica, apresentando os resultados parciais do período 2006 a 2008 , referentes aos dois primeiros níveis de avaliação - reação e aprendizado -, segundo o modelo de David Kirkpatrick (Kirkpatrick e Kirkpatrick, 2006).

\section{Metodologia}

Ao ser estruturado, o Programa de Capacitação Profissional em Biossegurança (PCPB) do Instituto Oswaldo Cruz (IOC) procurou aperfeiçoar o conteúdo com aprendizado. Nesse sentido, buscou atender as premissas diagnosticadas como itens fundamentais de Costa e Costa que afirmam:

O processo educacional deve refletir a cumplicidade entre professores, alunos e direção da instituição, visando a um "currículo motivador", onde os elementos significativos, como conteúdos, interações, contemporaneidade temática, entre outros, estejam devidamente contemplados (Costa e Costa, 2004, p. 49).

A figura 1 mostra um diagrama representativo dos ciclos básicos do planejamento do Programa de Capacitação Profissional em Biossegurança (PCPB). No primeiro ciclo, diagnóstico, foram realizadas duas pesquisas quantitativas através dos interlocutores de biossegurança (representantes dos 69 laboratórios de pesquisa do IOC). A primeira pesquisa teve como objetivo definir a real demanda, o interesse dos profissionais em participar do programa de capacitação e os macrotemas a serem abordados durante a capacitação, sendo informado o número de profissionais (de nível médio e superior) interessados em participar de cursos voltados para segurança laboratorial. A seguir, a segunda etapa visou coletar informações que subsidiassem o planejamento do conteúdo a ser abordado.

No segundo ciclo do planejamento, a partir dos dados coletados durante o diagnóstico, foi definido e implantado o PCPB, quais os profissionais que o programa atenderia e como seria a sua programação (conteúdo, carga horária, professores, material instrucional, entre outros itens), desde a sua estrutura até a sua avaliação. 


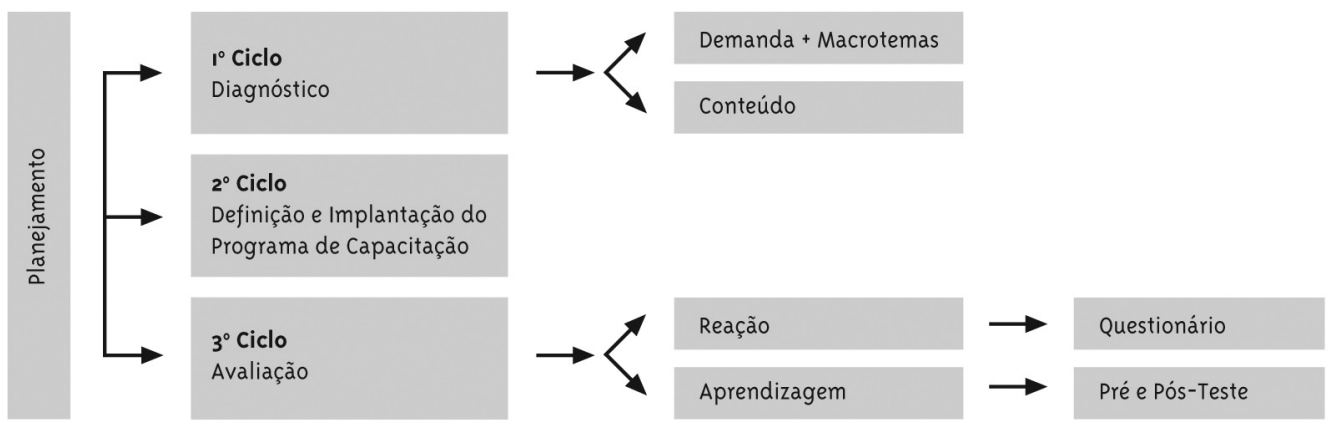

Para o ciclo de avaliação do Curso de Biossegurança para Laboratórios de Pesquisa Biomédica do Instituto Oswaldo Cruz, foi utilizado o modelo de análise de David Kirkpatrick (Evaluating Training Program, 1998), que se fundamenta em quatro níveis: (i) reação, realizada através de formulários, que mede as impressões dos participantes; (ii) aprendizado, estruturada através de testes, exames, simulações e seminários, que visa verificar se os participantes melhoraram ou ampliaram seus conhecimentos; (iii) comportamento, ou seja, a transferência dos novos conhecimentos e habilidades para o comportamento no trabalho; (iv) resultados, que pretendem determinar o impacto institucional (referendado por Bastos, 1994; Eboli, 2004). Neste artigo, foram avaliados os níveis reação e aprendizado, os quais discutiremos a seguir. As análises dos níveis comportamento e resultados estão em desenvolvimento dentro do escopo da pesquisa.

Para a avaliação da reação, ao final de cada módulo, formulários foram aplicados para que o profissional avaliasse o curso, no sentido de mensurar a satisfação dos participantes (com relação ao conteúdo, metodologia, instalações, material didático distribuído, entre outros). Para tanto, foi montado um questionário com questões fechadas, em que o profissional pôde opinar sobre o conteúdo, a postura dos professores, as instalações, a carga horária, a qualidade do material didático fornecido (livro e apostilas) pontuando: 1 (Ruim); 2 (Regular); 3 (Bom) e 4 (Excelente). 0 profissional pôde, ainda, apresentar suas críticas e sugestões em texto livre.
Para a avaliação do aprendizado, foram aplicados pré e pós-testes, no primeiro e no último dia de cada módulo, respectivamente, e as respostas foram computadas pelo coordenador e sua equipe, que verificaram o número de acertos de cada questão, procurando estabelecer, assim, o conhecimento prévio da turma. Essas informações foram repassadas aos professores que puderam ajustar os conteúdos de suas aulas, revendo a abordagem programada, de forma que o material de ensino fosse potencialmente significativo (Moreira, 1998).

\section{Resultados e Discussão}

No ciclo do diagnóstico realizado, com participação dos interlocutores, foram recebidos 36 questionários (52\% do total de interlocutores), que apontaram cinco macrotemas e revelaram o grande interesse de ambas as categorias profissionais (níveis médio e superior) em fazer parte do PCPB. As temáticas preferenciais são apresentadas na tabela 1, sendo biossegurança e boas práticas de laboratório as mais requisitadas, totalizando o interesse de $177 \mathrm{e}$ 209 profissionais, respectivamente.

Para Bastos (1994), na fase de diagnóstico é possível definir com clareza o problema a ser tratado, a necessidade a ser satisfeita, o objetivo a ser atingido por meio da capacitação. Essa fase evita o "treinar por treinar”. O diagnóstico, também chamado de avaliação de contexto (Depresbiteris, 2004), não se trata de uma tarefa isolada e formal, mas permite identificar o perfil dos participantes do programa, 
as competências que se pretende desenvolver.

A segunda pesquisa foi realizada visando gerar subsídios para o planejamento do conteúdo programático a ser abordado, de forma que pudesse ser significativo, atualizado, e que estivesse alinhado aos objetivos institucionais, respeitando as limitações de tempo e recursos disponíveis. Dessa forma, em cada temática, os interlocutores puderam opinar se o conteúdo era: 1 - importante e indispensável; 2 - não aplicável às atividades do laboratório; 3 - revisão completa, necessário ser enfatizado; 4 - excessivo, necessário reduzir. Para exemplificar, apresentamos, na tabela 2, parte do conteúdo programático da macrotemática "Boas Práticas de Laboratório", criteriosamente avaliado pelos interlocutores.

Considerando os dados coletados, na fase de planejamento foi definido que o PCPB seria subdivido em dois projetos, e esses seriam desenvolvidos concomitantemente. 0 primeiro projeto esteve voltado para os profissionais de nível médio em parceria com a Escola Politécnica de Saúde Joaquim Venâncio (EPSJV) da Fiocruz, que oferece o curso Boas Práticas de Laboratório de Saúde Pública (18o h/a). O segundo projeto deu origem ao Curso de Biossegurança para Laboratórios de Pesquisa Biomédica, destinado aos profissionais de nível superior, o qual foi programado levando em consideração os seguintes aspectos:

- Estrutura modular realizada ao longo do ano, com intervalo entre um módulo e outro, para que os participantes pudessem interagir e aplicar o que aprendessem no dia a dia no laboratório.

- Aulas com fortes conteúdos conceituais e normativos, exposição de vivência e experiência dos professores, estudo de casos e apresentação de seminários. Nessa situação, seria estimulada a participação dos profissionais que relatariam situações-problema identificadas nos seus laboratórios, tornando as aulas dinâmicas.

- A seleção e a identificação de competências institucionais para ministrar as aulas, levando em consideração não só o currículo pessoal de cada professor, mas também o conhecimento e a prática nos assuntos a serem abordados. Os professores externos seriam orientados sobre o foco, os objetivos e a estratégia do curso.

- Lideranças educadoras, com o envolvimento e comprometimento da alta administração (diretoria e chefias de laboratório), não só viabilizando financeiramente o programa de capacitação, mas atuando como agentes formadores e disseminadores na consolidação e transformação da cultura institucional. Nesse caso de biossegurança, como o alicerce para a construção do ideal organizacional almejado e proposto por Eboli (2004).

- Seleção de livros didáticos a serem distribuídos ao longo do curso para compor um acervo técnico no laboratório à disposição para consulta dos pesquisadores, técnicos e alunos.

- Curso presencial, voltado para o público interno, abrangendo todos os níveis hierárquicos (gerentes, pesquisadores, técnicos e alunos), realizado durante o horário de trabalho (meio período).

- Avaliação de resultado (descaracterizada da função seletiva, eliminatória e classificatória), no sentido de verificar se os objetivos educacionais foram atendidos. Além de possibilitar que em cada ciclo - planejamento-desenvolvimento-avaliação-fossem identificadas oportunidades de melhorias e ajustes que pudessem garantir a qualidade do programa e sua consequente longevidade.

Em seguida, foi possível estruturar o Curso de Biossegurança em Laboratórios de Pesquisa Biomédica, contemplando os módulos: introdutório (pré-requisito para os demais); riscos químico, físico e biológico; gestão da qualidade e experimentação animal. Também foram definidas as lideranças educadoras, pelos critérios descritos acima, e os livros didáticos de acordo com o tema do módulo. Em função do quantitativo de inscritos (nas turmas de 2006/2007/2008 foram capacitados 315 profissionais que atuavam em laboratórios de pesquisa do IOC, sendo $32,70 \%$ servidores), as aulas foram ministradas num auditório, contando com modernos recursos audiovisuais.

Ao término de cada módulo, segundo Kirkpatrick e Kirkpatrick (2006), foram realizadas as avaliações visando aferir a reação (o aluno avaliando o curso), no sentido de mensurar a satisfação dos participantes (com relação ao conteúdo, metodologia, instalações, material didático distribuído, entre outros). No período 2006 a 2008, 54,03\% dos profissionais declararam que o curso foi excelente; $39,59 \%$ classificaram o curso como bom e os demais $6,38 \%$ 
acharam que o curso foi regular ou não opinaram. Levando em consideração as propostas de melhorias apresentadas pelos profissionais e de forma a tornar as aulas mais dinâmicas, a coordenação do PCPB tem procurado desenvolver material didático específico para o curso, como o jogo de mímica "Biossegurança Divertida”, onde são reforçadas as condutas laboratoriais, caça-palavras e palavras cruzadas.

Na avaliação do aprendizado (o curso avaliando o aluno) foram realizados, a cada módulo, pré e póstestes, cujos resultados de 2006-2008 encontram-se detalhados na tabela 3.

No período 2006-2008, todos os módulos tiveram acréscimos nas médias do pós-teste em relação ao pré-teste, sendo os destaques o módulo físico em 2006 (31,03\%) e o módulo introdutório em 2007 e 2008 (32,63\%; 23,80\%, respectivamente). Interessante ressaltar que o Instituto Oswaldo Cruz atua nas áreas de pesquisa, desenvolvimento tecnológico e inovação e na prestação de serviços de referência para diagnóstico de doenças infecciosas e genéticas e controle de vetores. Assim, os conceitos e princípios de contenção relativos aos riscos biológicos e químicos, bem como para as atividades vinculadas à experimentação animal, foram significativamente apreendidos, apresentando médias nos pré-testes superiores a 72, com pequeno percentual de acréscimo com relação às médias dos pós-testes, o que comprova a familiaridade dos conteúdos de alguns módulos pelos profissionais. Já no módulo de risco físico - que contempla as questões relacionadas ao ruído, radiação, vibração, pressão, temperatura e iluminação -, foram observadas as menores médias nos pré e póstestes, o que evidencia como essa temática deve ser mais explorada, exigindo investimento em palestras e seminários que complementariam a capacitação profissional. Acreditamos que esses resultados estejam relacionados com as avaliações da reação dos alunos, no tocante ao critério "pertinência ou não dos temas abordados". Lacerda e Abbad (2003) chamam a atenção que a "percepção de utilidade" do curso influencia as avaliações de reação (afetivo) e aprendizagem (cognitivo). Segundo Lindeman (1926, apud Knowles e col., 2009), os adultos são motivados a aprender conforme percebem que a aprendizagem os ajudará a executar tarefas ou lidar com problemas que vivenciam em sua vida. Assim, entendemos que quanto maior a discussão dos temas e negociação dos significados (Lemos, 2006), maior será a possibilidade de ocorrência da aprendizagem.

Através dessa avaliação, que não se trata de uma avaliação autoritária, classificatória e seletiva, que exige um controle e enquadramento dos indivíduos (Luckesi, 2006), uma vez que a certificação é dada em função da frequência ( $75 \%$ ), o profissional tem maior clareza sobre sua aprendizagem, tem autonomia para decidir o que estudar, podendo até mesmo participar novamente do módulo no ano seguinte, caso não obtenha bons resultados, para rever os conceitos, aprofundar seus conhecimentos e dirimir dúvidas. Essa avaliação objetiva, como citado por Ausubel (um dos principais autores da Teoria da Aprendizagem Significativa), promover a aprendizagem em sala de aula, verificando os conceitos que o aprendiz já conhece antes da efetivação do ensino, além de acompanhar e aperfeiçoar a evolução da aprendizagem e verificar se os objetivos foram alcançados, assim como se as estratégias utilizadas foram as mais eficazes e apropriadas (Lemos, 2006). Além disso, ela também permeia todo o processo de conhecimento, subsidiando as decisões dos docentes, seja na questão do planejamento, continuidade, ou mesmo reestruturação do conteúdo, recursos e estratégias adotadas (Lemos, 2006).

É comum o caso de profissionais que avaliam muito positivamente o curso (reação), que demonstraram que aprenderam (aprendizado), mas não alteraram seu comportamento na situação do trabalho. Por isso, as avaliações dos níveis comportamento e resultados são tão importantes, e estão em andamento para a sua efetiva implementação pela coordenação do PCPB.

Entretanto, de modo indireto, podemos observar uma mudança no comportamento do profissional apontando algumas situações interessantes, como o aumento (i) no consumo (21,46\% nos últimos seis meses) de equipamentos de proteção individual, como jalecos descartáveis, luvas de procedimento, luvas nitrílicas e máscaras; (ii) no pedido de credenciamento, em relação à biossegurança, dos laboratórios do IOC visando à manipulação de organismos geneticamente modificados, em consonância com a Lei 11.105/05 (iii) na rapidez com que as vagas ofe- 
recidas nos cursos foram preenchidas. Em 2008, as 110 vagas oferecidas esgotaram em menos de uma hora (Soeiro e Pereira, 2009).

\section{Considerações Finais}

Atualmente, vive-se a transição da sociedade moderna para a sociedade do conhecimento, onde as mudanças são extremamente velozes e exigem uma constante atualização. No Brasil, algumas empresas evoluíram e cresceram com base em programas de capacitação bem concebidos e estruturados, sendo um grande desafio a criação de indicadores eficazes de mensuração dos resultados obtidos com os investimentos realizados (Eboli, 2004).

O Projeto de Modernização da Gestão Científica do Instituto Oswaldo Cruz foi estruturado exatamente para atender essa premissa, que é uma necessidade natural das organizações de Ciência, Tecnologia e Inovação (CT\&I) no atual cenário de competição crescente, que buscam assegurar a disponibilização, integração e divulgação do conhecimento, utilizando como estratégia, principalmente, a capacitação (IOC, 2007).

O PCPB do IOC visa gerar mudanças de comportamento (transmissão de conhecimentos, desenvolvimento de habilidades, alteração de atitudes), buscando uma nova postura de trabalho. 0 profissional deve ser responsável não só por sua própria segurança, mas também pela do seu colega e do ambiente. É fundamental que ele perceba que faz parte de uma rede de relacionamento, em que os elos são sempre mais fortes quando em conjunto. Para tanto, o programa de capacitação deve espelhar a real necessidade de seus participantes.

Dessa forma, torna-se indispensável o levantamento de necessidades, a definição de objetivos, planejamento, implantação e, por último, a avaliação de resultados; uma vez que o monitoramento permite obter informações para aperfeiçoar e comprovar a relação custo-benefício vantajosa para a instituição. O modelo de Kirkpatrick (Kirkpatrick e Kirkpatrick, 2006), adotado neste estudo, mostrou-se extremamente eficaz por possibilitar diferentes tipos de avaliação, sendo imprescindíveis para uma visão globalizada de processo de aprendizado em biossegurança numa instituição de Ciência e Tecnologia para a saúde. Percebemos que, ao aplicarmos a proposta sugerida por Kirkpatrick, os resultados encontrados nos apontaram estratégias a serem seguidas no aperfeiçoamento desse modelo de educação continuada em biossegurança.

\section{Referências}

AMORIN, M. C. S. Comunicação planejada, recursos fundamental para a eficácia da gestão organizacional. Caderno de Pesquisa em Administração da USP, São Paulo, v. 1, n. 9, p.98108, 1999. Disponível em: < http://www.ead.fea. usp.br/cad-pesq/arquivos/c9-Art8.pdf >. Acesso em: 12 maio 2010.

BARROS JR, B. L.; SILVA, A. S. Biossegurança e qualidade de vida em atividades agropecuárias de produção e ensino. Cadernos Temáticos, Brasília, DF, n. 6, p. 38-43, 2005.

BASTOS, O. P. M. Diagnóstico e avaliação de T\&D: processo de T\&D. In. BO0G, G. G. (Org.). Manual de treinamento e desenvolvimento ABTD. São Paulo: Makron Books, 1994. p. 137-163.

COSTA, M. A. F. et al. Biossegurança no ensino médio: uma discussão preliminar sobre conteúdos em livros didáticos de ciências e práticas docentes. In: SEMINÁRIO NACIONAL DE EDUCAÇÃO PROFISSIONAL E TECNOLÓGICA, 1., 2008, Belo Horizonte. Disponível em: <http:// www.senept.cefetmg.br/galerias/Arquivos_senept/ anais/terca_tema1/TerxaTema1Artigo3.pdf>.

Acesso em: 12 maio 2010.

COSTA, M. A. F.; COSTA, M. F. B. A biossegurança na formação profissional em saúde: ampliando o debate. In: PEREIRA, I. B.; RIBEIRO, C. G. (Org.). Estudos de politecnia e saúde. Rio de Janeiro: Escola Politécnica de Saúde Joaquim Venâncio, 2007. p. 253-272.

COSTA, M. A. F.; COSTA, M. F. B. Educação e competências em biossegurança. Revista Brasileira de Educação Médica, Rio de Janeiro, v. 38, n. 1, p. 46-50, 2004.

DEPRESBITERIS, L. Instrumentos y técnicas de evaluación de impactos y aprendizaje en programas educativos de capacitación laboral: la necesidad de conjugación. Estudos em Avaliação Educacional, São Paulo, n. 29, p. 5-48, 2004. 
EBOLI, M. Educação corporativa no Brasil: mitos e verdades. São Paulo: Editora Gente, 2004.

FLEURY, A.; FLEURY, M. T. L. Aprendizagem e inovação organizacional: as experiências de Japão, Coréia e Brasil. São Paulo: Atlas, 2006.

FUNASA - FUNDAÇÃO NACIONAL DE SAÚDE. Fiocruz preparada para enfrentar doença que atinge Ásia, Europa e América do Norte. Brasília,, 2003. Disponível em: <http://www.funasa.gov. br/Web\%2oFunasa/not/not2003/not406.htm>. Acesso em: 22 ago. 2008.

FUNDAÇÃO OSWALDO CRUZ. Instituto Oswaldo Cruz. Comissão Interna de Biossegurança - CIBio. Relatório de atividade. Rio de Janeiro, 2007.

GIR, E. et al. Biossegurança em DST/AIDS: condicionantes da adesão do trabalhador de enfermagem às precauções. Revista da $E$ scola de Enfermagem da USP, São Paulo, v. 38, n. 3, p. 245253, 2004 .

IOC - INSTITUTO OSWALDO CRUZ. A caminho do novo. Informe do IOC, Rio de Janeiro, v. 13, n.7, 2007. Disponível em: <http://www.ioc.fiocruz.br/pages/ informerede/corpo/informeemail/2007/1304/ principal1704.html>. Acesso em: 23 ago. 2008.

KIRKPATRICK, D. L.; KIRKPATRICK, J. D.

Evaluating training programs: the four levels. San Francisco: BK, 2006.

KNOWLES, M. S.; HOLTON III, E. F.; SWANSON, R. A. Aprendizagem de resultados: uma abordagem prática para aumentar a efetividade da educação corporativa. Rio de Janeiro: Elsevier, 2009.

LABARTHE, N. V.; PEREIRA, M. E. C. Biossegurança na experimentação e na prática da clínica veterinária. Ciência Veterinária nos Trópicos, Recife, v. 11, p. 153-157, 2008. Suplemento 1.

LACERDA, E. R. M.; ABBAD, G. Impacto do treinamento no trabalho: investigando variáveis motivacionais e organizacionais como suas preditoras. Revista de Administração Contemporânea, Curitiba, v. 7, n. 4, p. 77-96, 2003.

LEMOS, E. S. Aprendizagem significativa: estratégias facilitadoras e avaliação. Série Estudos:Periódicos do Mestrado em Educação do $U C D B$, Campo Grande, n. 21, p. 53-66, 2006.
LUCKESI, C. C. Avaliação da aprendizagem escolar: estudos e proposições. 18. ed. São Paulo: Cortez, 2006.

MARINHO, C.; MINAYO-GOMES, C.; DEGRAVE, W. Qualificação e percepção de riscos de trabalhadores da área biotecnológica: setores público e privado. Caderno CRH, Salvador, n. 32, p. 259-278, 2000.

MASTROENI, M. F. A difícil tarefa de praticar a biossegurança. Ciência e Cultura. São Paulo, v. 6o, n. 2, p. 4-5, 2008.

MOREIRA, M. M. Mapas conceituais e aprendizagem significativa. Cadernos de Aplicação, Porto Alegre, v. 11, n. 2, p. 143-156, 1998.

PEREIRA, M. E. C.; BORBA, C. M.; JURBERG, C. Gestão de biossegurança: o papel das comissões internas de biossegurança. In: COSTA, M. A. F.; COSTA, M. F. B. (Org.). Biossegurança geral para cursos técnicos da área de saúde. Rio de Janeiro: Publit, 2009b. p. 301-308.

PEREIRA, M. E. C.; BORBA, C. M.; JURBERG, C. O papel da Comissão Interna de Biossegurança: a experiência do Instituto Oswaldo Cruz. Revista Eletrônica de Comunicação, Informação e Inovação em Saúde, Rio de Janeiro, v. 3, n. 4, p. 226-233, 2009a.

PORTO, M. F. S.; FREITAS, C. M. de. Análise de riscos tecnológicos ambientais: perspectivas para o campo da saúde do trabalhador. Cadernos de Saúde Pública, Rio de Janeiro, v. 13, p. 59-72, 1997. Suplemento 2.

SOEIRO, M. N. C.; PEREIRA, M. E. C. Programa de capacitação em biossegurança do Instituto Oswaldo Cruz: o impacto na qualidade de vida do profissional. In: COSTA, M. A. F.; COSTA, M. F. B. (Org.). Biossegurança geral para cursos técnicos da área de saúde. Rio de Janeiro: Publit, 2009. p. 358-371.

Recebido em: 03/08/2009

Reapresentado em: $11 / 01 / 2010$

Aprovado em: 12/01/2010 